\title{
LA EVALUACIÓN COMO INSTRUMENTO PARA MEJORAR LA CALIDAD EDUCATIVA. Reflexiones sobre las últimas tendencias en los procesos de evaluación
}

\author{
Guadalupe MéndezZamalloa,Ph.D.
}

\begin{abstract}
RESUMEN
La evaluación como medio de diagnóstico, desarrollo y valoración de acciones educativas merece, actualmente, gran atención de los académicos y prácticos del campo de la educación. Así, lo demuestra este artículo, al analizar la evaluación de los aprendizajes desde una perspectiva moderna enfatizando la auto y coevaluación, como el uso de instrumentos no convencionales de evaluación. La evaluación del docente es ampliamente tratada a la luz de los últimos desarrollos en el país y se concluye con la evaluación institucional, especialmente dirigida a la acreditación.
\end{abstract}

\section{ABSTRACT}

The evaluation as an instrument of diagnosis, development and appraisal of educational actions currently needs to be taken into consideration by professors and academics of the education field. This article analyses the assessment of learning from a modern view emphasizing the auto evaluation and co evaluation, as well as the use of non-conventional evaluation instrLme nt $\mathrm{s}$. The evaluation of teachers is widely analyzed, considering the latest developments in the country and the institutional evaluation, particularly appointed tothe accreditation.

\section{PALABRAS CLAVE}

Evaluación, evaluación de los aprendizajes, evaluación del docente, evaluación institucional, ámbitos de la evaluación, metacognición.

\section{KEYWORDS}

Evaluation, learning evaluation, evaluation of the teacher, institutional evaluation, evaluation areas, metacognition.
ए n los últimos años, tanto en el Perú como a nivel mundial, se ha generado una intensa discusión sobre el mejoramiento de la calidad de la educación, sobr la necesidad de que los aprendizajes de los alumnos sean relevantes y respondan al desarrollo de la sociedad, de que éstos concurran con los adelantos científicos y tecnológicos y que las nuevas generaciones estén preparadas para crear, producir e innovar y, consecuentemente, se puedan integrar fácilmente en este mundo global donde la tecnología y el conocimiento crecen a pasosagigantados. El temaes que la educación debe asegurar la formación de ciudadanos para que participen plenamente en la vida política, social y económica de la sociedad. Todas, deuna forma u otra, sabemos quela educación es la base del desarrollo, que es un derecho indiscutible del ser humano y que sin una educación de calidad difícilmente la sociedad alcanzará las metas que aspiran los diferentes países.

En la preocupación de mejorar sus sistemas educativos, los países que tienen los mejores sistemas educativos trabajan por seguir siendo los primeros; otros, que tienen una alta performance, lo hacen para alcanzar la excelencia, y aquellos que tienen problemas, para hacerlo bien, se esfuerzan por identificar y alcanzar aquellas características que distinguen a los mejores sistemas educativos.

El gran detonante para esta saludable preocupación por la calidad educativa ha sido los resultados de las pruebas de evaluaciones internacionales $y$, en algunos casos, nacionales aplicadas en muchos países. Los resultados de las pruebas PISA estremecieron a más de un país. Esta prueba es 
aplicada por la Organización para la Cooperación y el Desarrollo Económico (OCDE) cada tres años desde el año 2000. Esta organización establece un nivel promedio de logro para cada una de las áreas que evalúa, el mismo que sirve de medida comparativa de loslogros alcanzados por los países participantes.

El año 2000 participaron en esta prueba 41 países. Desde entonces muchos países de los diferentes continentes han ido incorporándose paulatinamente a esta evaluación. Argentina, Chile, Brasil, México y Perú participaron en estas pruebas. Respecto al rendimiento del estudiante, los resultados para estos países no fueron satisfactorios. En el ranking final, Argentina ocupó la posición 33, México la 35, Chile la 36, mientras que Brasil estuvo en el lugar 37 y Perú ocupó el último lugar. Perú no participó de las pruebas aplicadas los años 2003y 2006.

En el 2009, sesenta y cinco sistemas educativos participaron en la prueba global de desempeño estudiantil que evalúa las habilidades de jóvenes ele 15 años en matemática, lectura y ciencias. Finlandia y Singapur ocuparon, nuevamente, los primeros lugares. En esta oportunidad participaron nueve países de Latinoamérica: Argentina, Brasil, Chile, Colombia, México, Panamá, Perú, Triniclad y Tobago y Uruguay. Los resultados publicados este año señalan que algunos países de Latinoamérica y el Caribe mejoraron su desempeño, aunque ninguno alcanzó los niveles elela OCDE.

Asimismo, el Ministerio de Educación del Perú implementa desde el año 2007 la Evaluación Censal de alumnos ele 2do. grado ele primaria ele escuelas públicas y privadas en las áreas de comprensión lectora y matemática. Los resultados desalentadores de estas pruebas han ido mejorando con bastante lentitud, a pesar de las reformas pedagógicas emprendidas como la implementación del plan lector, el aumento de las horas de matemática, el PELA (Programa estratégico de logros del aprendizaje): el mismo que concentra su atención en los niños y niñas de Educación Inicial de 3 a 5 años y elelos dos primeros grados dela Educación Primaria.

La evaluación del año 2010, cuyos resultados han sido publicados por el Ministerio de Educación a inicios de este año, presenta información más gratificante, aunque todavía con serias preocupaciones y demandas. Se señalan logros a nivel nacional de 28,7\% en Comprensión Lectora y 13,8\% en Matemática. El análisis de los resultados muestra marcadas diferencias de logros de aprendizaje entre las escuelas urbanas y rurales; las diferencias entre las escuelas públicas y privadas, así como el menor rendimiento en el área de matemática, tanto en las escuelas públicas como privadas en relación a los de comprensión lectora. Es en esta área donde existe la mayor brecha entre las escuelas urbanas y rurales. Estos resultados nos envían importantes mensajes, ratifican una vez más la necesidad de atender en forma equitativa a todos los niños y niñas de las diversas regiones del Perú y, muy especialmente, a los de las zonas rurales y de extrema pobreza, así como la necesidad de prestar mayor atención a la formación delos estudiantes en el área delas ciencias.

Países como el Perú y otros que no han salido bien en las pruebas PISA, LLECE, o en evaluaciones nacionales en las cuales se han demostrado bajos rendimientos, han venido promoviendo e implementando medidas pedagógicas orientadas al mejoramiento de los aprendizajes en las áreas evaluadas, como reformas de contenidos curriculares, capacitación docente, mayor número de horas, etc. Algunos cambios educativos en estos países han respondido a planes educa tí vos articulados a los resultados de estas evaluaciones, a las exigencias de la sociedad, o a la presión y exigencia de los propios estudiantes por una educación de calidad, como hace poco se ha observado en las manifestaciones públicas en el vecino país de Chile.

Lo cierto es que los resultados de las evaluaciones internacionales y nacionales de los estudiantes nos han hecho ver con claridad que no se está prestando suficiente atención, no solo a los resultados educativos, sino también a los procesos y a las condiciones en que éstos se promueven. Esta situación ha determinado claramente la necesidad de contar con sistemas de evaluación articulados y orientados a la toma de decisiones para mejorar la calidad educativa.

Sin lugar a dudas el impacto de la evaluación en la calidad del aprendizaje es significativo, como lo demuestra un gran número de investigaciones desarrolladas. Creemos que son tres los procesos de evaluación que, implementados en forma oportuna, sistemática y responsable, pueden permitirnos avanzar en la ruta del mejoramiento de la ansiada calidad educativa. Estos procesos están fundamentalmenteligados a los aprendizajes de los alumnos, a la labor del maestro y a la gestión de la institución educativa. El logro de resultados de aprendizajes de calidad por parte de los estudiantes es la razón del sistema educativo. El maestro y la escuela son los mediadores para que esto ocurra.

\section{1 un1Fé}


A continuación, una breve reflexión sobre cada uno de estos aspectos, solo con el objetivo de ver en forma global las nuevas tendencias que se vienen desarrollando dentro de estos procesos de evaluación en respuesta a la actual exigencia de la sociedad de contar con una educación de calidad .

El Proyecto Educativo Nacional al 2021 elaborado por el Consejo Nacional de Educación es una política de Estado que enmarca, delimita y orienta las políticas de gobierno en materia educativa, con el fin de asegurar que la educación cumpla su verdadero sentido y rol, que es la de proveer una educación para la realización de todos los peruanos y para la edificación colectiva de la democracia y desarrollo del país, asegurando la continuidad de los procesos de cambio en una perspectiva de largo plazo (PEN, 2007). Este proyecto, aprobado por Resolución Suprema y reconocido por el Acuerdo Nacional el año 2007, plantea seis grandes objetivos estratégicos. Elsegundo objetivo: Estudiantes einstituciones que logran aprendizajes de calidad; y el tercero: Maestros bien preparados que ejercen profesionalmente la docencia, guardan relación con el propósito de este artículo, y sirven de marco para la reflexión en torno a los procesos de evaluación señalados.

\section{Evaluación de los aprendizajes de los alumnos}

Sabemos que la evaluación es un proceso participativo, reflexivo, crítico, formativo e integral, basado fundamentalmente en el desempeño del alumno y en la aportación de evidencias o productos. En los círculos docentes se ha discutido con amplitud el concepto de evaluación, los momentos de la evaluación durante el proceso de enseñanzaaprendizaje, así como las técnicas e instrumentos a ser utilizados en concordancia con las competencias a ser evaluadas.

Es totalmente reconocido que la construcción de los elementos de evaluación responde a lo que se desea alcanzar, a fin de que el docente pueda tomar decisiones para mejorar los procesos pedagógicos y responder a los criterios de desempeño seleccionados. Está claro también que la evaluación debe ser planificada como parte del proceso enseñanza -apren dizaje y no como una situación aislada. Sabemos del uso de un sinnúmero de estrategias evaluativas diseñadas, organizadas y aplicadas por el maestro dentro del aula. Sin embargo, cuando observamos el entorno de la escuela, encontramos que aún cuando existen innovaciones en las estrategias de aprendizaje en muchísimos centros educativos, éstas están acompañadas de sistemas de evaluación tradicionales.

La práctica evaluativa de los aprendizajes sigue en debate. Esta pareciera haberse focalizado, fundamentalmente, en el uso de determinadas técnicas e instrumentos utilizados por el profesor para evaluar a sus alumnos teniendo en consideración que la experiencia educativa solo se produce en el aula, empleando procedimientos iguales entre desiguales y con un especial énfasis en los resultados conseguidos. ¿Son estas prácticas equivocadas? En absoluto. Lo que creemos es que tienen que ser reorientadas y complementadas para ver a la evaluación como un proceso de reflexión pedagógica sobre lo que se hace, cómo se hace y qué utilidad tiene.

Nuevas propuestas evaluativas que responden a corrientes pedagógicas de aulas abiertas se vienen utilizando cada vez con mayor énfasis, donde los aprendizajes no se desarrollan solo en el aula sino también fuera de ella, en diferentes escenarios, con diferentes propuestas a fin de que los estudiantes asuman retos cognitivos. Existen nuevas estrategias y técnicas evaluativas que son en sí mismas estrategias para el aprendizaje y, a su vez, contenidos de aprendizaje. Es, en realidad, una nueva forma de pensar y hacer evaluación. No existen momentos de evaluación, ni finales, ni paralelas, sino evaluaciones integradas al aprendizaje mismo, estrategia s que permiten al alumno aprender y al mismo tiempo evaluar su aprendizaje $\mathrm{y}$, por consiguiente, tomar decisiones para su mejor desarrollo.

Este enfoque socio-cognitivo, denominado también de evaluación auténtica, representa un gran avance en el campo de la evaluación y responde de manera global a las exigencias de corrientes pedagógicas cognitivas, exigencias de la sociedad actual, cambiante y global, a las necesidades de inclusión y atención a las diferencias, así como al requerimiento de generar acuerdos y consensos sobre esta práctica entre las partes que intervienen en el proceso educativo, es decir maestros y alumnos. El punto de partida de este enfoque es la concepción constructivista del aprender, reconociendo que el conocimiento yla regulación delos propios procesos cognitivos sonlaclave parafavorecerlacapacidad de aprender a aprender . Esta nueva visión postula que la docencia, más que transmitir saberes constituidos y legitimados socialmente, debería responsabilizarse por asegurar las condiciones óptimas para que los alumnos desarrolled sus capacidades cognitivas, afectivas y sociales que les permitan construir sus 
aprendizajes. Ya no se espera tanto que los profesores causen el aprendizaje en sus alumnos sino que éste sea causado por los propios alumnos, que, por lo mismo, puedan ser ellos responsables de su propio quehacer.

El desarrollo de la capacidad de aprender a aprender exige nuevos planteamientos en la evaluación, estrategias que estimulen las habilidades metacognitivas para que el estudiante tome conciencia de su propio proceso de aprendizaje, de sus avances, estancamientos, de las acciones que le han hecho progresar y de aquellas que le han inducido al error. Por lo tanto, la evaluación se convierte entonces en un instrumento de aprendizaje, que ayuda al estudiante a tomar conciencia de los aprendizajes logrados, de los procesos desarrollados en la adquisición deéstosyde cómo regularlos.

Por otro lado, así como la educación tiene que adaptarse a una sociedad de acelerados avances científicos y tecnológicos y de profundos cambios en el ámbito profesional y social, la formación de los estudiantes debe considerar el desarrollo de habilidades específicas apropiadas para enfrentar estos cambios, incluyendo determinadas actitudes y valores. Esto solo se logra con una acción formativa constante y también a través de una evaluación continuada que toma en cuenta, no solo los procesos formalizados de enseñanza-aprendizaje (en el aula), sino todas aquellas situaciones que favorecen la formación, planificadas o no. Toda persona debe estarencapacidad devalorarsuactividadformativa a lo largo de la vida. Queda claro que las nuevas generaciones necesitan herramientas para autoevaluarse ysaber evaluar.

Este enfoque de evaluación fomenta la autoevaluación y la ca-evaluacióncon la finalidad de que sean los estudiantes quienes valoren sus logros en las diferentes áreas. El docente también evalúa pero con fines de retroalimentación; es decir, con la finalidad de orientar a sus estudiantes en el proceso de enseñanza-aprendizaje utilizando múltiples técnicas e instrumentos de evalua ción . La autoevaluación permite al estudiante evaluar su propio desempeño, emitir juicios sobre sí mismo, participar de manera crítica en la construcción de su aprendizaje y retroalimentarse constantemente. La ca-evaluaciónes una valoración conjunta sobre la actuación del grupo. A través de ella se evalúa los logros personales y grupales, se fomenta la participación, reflexión y crítica; es el grupo de estudiantes quien se evalúa entre sí.
Con la autoevaluación y la ca-evaluación los estudiantes desarrollan una mayor autonomía y autoconciencia sobre su aprendizaje, les permite desarrollar la capacidad de identificar lo que saben y lo que les falta. Estas son formas de evaluación significativa, dentro del proceso de aprendizajes significativos, que permite a los estudiantes autoregular susaprendi zajes.

Teniendo en cuenta los procesos reflexivos que el estudiante realiza tanto en su autoevaluación como en la evaluación de sus pares, podemos señalar que los aprendizajes que logra vienen de sí mismo, que el aprendizaje está garantizado porque surge del propio sujeto. Los errores que detecta el estudiante en su proceso de autoevaluación son el punto de partida para un proceso de auto -aprendi zaje. Es el propio alumno quien valora sus aciertos $\mathrm{y}$ desaciertos en el proceso de aprendizaje, mejora sus resultados y habilidades cognitivas. En suma, esta es una evaluación formadora que viene desde dentro, va más allá de una evaluación formativa centrada en la intervención delmaestro.

Por lo expuesto, se puede concluir que este nuevo enfoque de la evaluación de los aprendizajes enfatiza cuatro características: 1 ) una evaluación formadora que se fundamenta en el auto-aprendizaje; 2) una evaluación multicultural, que atiendelas diferencias personales, culturales, étnicas, religiosas y socioculturales; 3) una evaluación centrada en el aprendizaje y no en el control externo; 4) una evaluación participativa y consensuada que permite que el alumno forme parte de las decisiones evaluativas.

Desde esta perspectiva, creemos que las siguientes técnicas de evaluación, que son además estrategias de aprendizaje, deben ser tomadas en cuenta.

Portafolio de aprendizaje: el estudiante incorpora evidencias significativas de producción donde se relatan de manera reflexiva los logros, progresos y dificultades en períodos y áreas específicas. El uso de este instrumento permite al estudiante crecer y mejorar tanto en la parte académica como personal. Esta es una técnica importante de la autoevaluación que exige autodisciplina y responsabilidad por parte del alumno. Se utiliza también como medio de evaluación externa de la labor docente.

Diario reflexivo: instrumento de análisis y reflexión que se trabaja con los estudiantes para que éstos identifiquen sus logros, ya sea

\section{1s I un1Pé}


individual o grupalmente. A modo de diario personal, refleja la experiencia de aprendizaje de una unidad o momento determinado del proceso de aprendizaje. El estudiante hace un análisis y síntesis de lo aprendido, reflexiona sobre el mismo, y escribe sus dudas, sentimientos y actitudes experimentadas, etc. Es una excelente estrategia evaluativa para desarrollar las actividades metacognitivas.

- Mapas conceptuales: son recursos gráficos que permiten representar jerárquicamente los conceptos y proposiciones sobre un tema determinado y las conexiones que ha logrado establecer entre ese conocimiento y otros que posee. Favorece el desarrollo organizado y funcional de los conceptos claves de una materia o disciplina. Esta es una estrategia cognitiva muy potente para evaluar el proceso de aprendizaje, el docente deberá establecer determinados criterios paravalorarlos.

- Contratos didácticos: que se concretan a través de un plan de acción negociado entre el alumno y el profesor. Este instrumento permite medir criterios de desempeño de la dimensión personal, como valorar la responsabilidad, el compromiso, la ética, la capacidad de metacognición del estudiante.

- Registros anecdóticos: fichas para recoger comportamientos, aportan información significativa para evaluar carencias o actitudes positivas; permiten seguir la evolución de una conducta o comportamiento en particular. El docente registra acontecimientos significativos y relevantes en relación a un estudiante, con el cuidado de no incluir opinión o interpretación de los hechos.

En resumen, cuando la evaluación es constructivista elalumno participa de las decisiones en el proceso de enseñan za-apre ndizaje, comprometiéndose con su aprendizaje, autoevaluándose y evaluando a sus compañeros y al proceso. El docente facilita el aprendizaje del alumno, promoviendo su participación y contribuyendo a su desarrollo integral, planteándole la evaluación como una actividad continua, integral y retroalimentadora. El profesor requiere de un conocimiento profundo de sus estudiantes, que sólo podrá obtener al considerar cuáles son sus necesidades, intereses, conocimientos previos, estilos de aprendizaje, motivaciones intrínsecas y extrínsecas, hábitos de trabajo, actitudes yvalores, entre otros aspectos.

\section{Evaluación docente}

Hemos señalado que la utilización de nuevos enfoques evaluativos permitirían mejorar los niveles de aprendizaje en los estudiantes; sin embargo, creemos que el elemento más importante de un sistema educativo para generar estos resultados es la calidad de la enseñanza. Es el maestro el primer responsable de esta tarea, el eje central del proceso educativo, el promotor de estos aprendizajes, aquel maestro que conoce a su alumno, se identifica con él y sabe cómo aprende.

Altos niveles de aprendizaje requieren de altos niveles de habilidades docentes. El Estado, comprometido con el crecimiento económico actual del país y el acceso de todos los ciudadanos a este crecimiento, afronta el desafío de fortalecer la profesión docente.

El problema de la docencia es uno de los factores que más afecta la calidad educativa en el Perú. Este se expresa en la deficiente calidad de la formación de algunos docentes, bajos niveles de desempeño, escasos e insuficientes niveles de ingreso y desarrollo de sus funciones dentro de un marco normativo que no incentiva su profesionalización. Dentro de este panorama, Icómo esperamos que estos maestros produzcan altos niveles de aprendizajes en sus alumnos? ¿Cuáles son las características de una buena docencia? ¿Cómo sabremos quiénes son los buenosmaestros? ¿Cómo preparamos a aquellos que no lo son para que sean buenos maestros? Por lo general, la definición de un buen docente varía de persona apersona, ya sea de acuerdo a su experiencia, percepción o formación. Se hace necesario, pues, articular, de manera objetiva y coherente, los elementos que constituyen una buena docencia, y luego desarrollar políticas tanto para la formación inicial de los docentes como para la evaluación de los maestros en ejercicio, que promuevan dicha docencia.

la historia del desarrollo de estos criterios tiene una larga data. Diversos han sido los factores identificados como ideales en la docencia, desde el énfasis en las características personales del maestro, hasta prácticas docentes más o menos rigurosas. Recientemente, los órganos oficiales educativos han venido examinando las políticas educativas de diferentes países en torno a la definición de una buena docencia y han observado que los países que exhiben mejores resultados académicos tienen una cultura muy profesic,ial con respecto a la docencia. 
En Finlandia, por ejemplo, cuyos estudiantes obtienen los mejores resultados en muchas de las pruebas internacionales, solo el $10 \%$ de quienes desean seguir la profesión docente son admitidos a los programas de licenciatura. la selección para el ingreso a la formación docente inicial es sumamente rigurosa. la consecuencia de estas políticas es que la docencia es una profesión de alto nivel, a la cual desean ingresar muchos de los egresados con mejores resultados académicos. En esta misma línea, en el Perú hace un par de años, se dispuso el ingreso a las instituciones de formación pedagógica con una nota mínima de14.

Como resultado de las demandas por una mejor calidad de la educación, muchos países han est ablecido diversas formas de evaluación del desempeño docente, incluyendo o no niveles de desempeño. los esquemas, métodos, criterios y estándares de evaluación docente vigentes en los sistemas educacionales adoptan múlt iple s formas y difieren entre ellos, algunos de los cuales se han basado en el Marco de desempeño docente de Danielson, C. (2007), quien establece cuatro dominios de responsabilidades del maestro: planificación, ambi ente en el aula, instrucción y responsabilidades profesionales, cada uno con diversos componentes. De la misma forma, muchos, como en el caso del Perú, han intentado vincular su sistema de evaluación docente con el reconocimiento, las mejoras económica s, así como las oportunidades de desarrollo profesional del maest ro.

Para nuestra satisfacción, dentro del magisterio nacional se ha internalizado en gran medida la cultura de la evaluación. Muchos maestros reconocen la necesidad de participar en procesos evaluativos que les permitan no solo su desarrollo personal y profesional, sino también la posibilidad de acceder a mejores ubicaciones profesionales, a elevar su economía y, por supuesto, a asegurar que sus alumnos logren aprendizajes de calidad. Existen también aquellos maestros que, influenciados por posiciones políticas, niegan totalmente las ventajas y oportunidades que los procesos de evaluación permiten. Algunos, que no quieren cambiar su zona de comodidad y se conforman con lo que son, con lo que tienen y con lo poco que ofrecen a sus alumnos .

Como se señaló con anterioridad, el Proyecto Educativo Nacional, a través de uno de sus objetivos estratégicos, plantea la urgente necesidad del país de contar con maestros bien preparados para ejercer profesionalmente la docencia. Pone énfasis en el desarrollo profesional docente y en su revaloración a través del ejercicio de la Carrera Pública Magisterial (CPM) centrada en un desempeño responsable. Una de las políticas, dentro de este gran objetivo, es evaluar a los docentes tanto para el ingreso y permanencia en la CPM, así como para su asignación laboral.

la Carrera Pública Magisterial , que se viene implementado en el Perú desde 2008, tiene como objetivo fundamental garantizar la calidad educativa, la idoneidad de los docentes y su buen desempeño para atender el derecho de cada alumno a un maestro competente. Promueve la meritocracia , es decir, el reconocimiento del esfuerzo y de los logros. Desafortunadamente, en nuestro país, la experiencia reciente de las evaluaciones a los maestros para acceder a la Carrera Pública Magisterial no fue totalmente gratificante. Problemas de gestión, logística, control, entre otros, así como problema s técnicos en la elaboración de las pruebas, crearon serias confrontaciones en tr e maestros, gremios docentes, el Es tado y la sociedad en general. El gran cuestionamiento, que se ha expresado con mucho énfasis, ha sido el qué de la evalua ción; es decir qué aspect os deben ser evaluados, qué características definen lo que es un buen docente, cuáles son los criterios de desempeño docente de referencia.

El Consejo Nacional de Educación (C NE), con el apoyo de la mesa interinstitucional, la misma que reúne al estado con la sociedad civil, ha venido trabajando este tema desde hace poco más de dos años . luego de una serie de consultas con diversos agentes de la sociedad, y dos congresos pedagógicos desarrollados, este organismo plantea una propuesta denominada Marco de Buen Desempeño Docente. Esta propuesta identifica criterios de desempeño docente que servirán, luego de revisiones y consensos para su legitimidad, como una base para delinear las políticas docentes para los próximos años; es decir, aquellas relacionadas con la formación inicial, la formación continua, y por supuesto, con la evaluación docente. Recientemente el Ministerio de Educación ha reconocido este esfuerzo y ha señalado, también, la necesidad de continuar con la implementación de la CPM, proceso que debe incorporarlosmecanismos yconsensos pertinentes.

la propuesta de Marco de Buen Desempeño Docente consta de 33 criterios categorizados en cuatro grandes dimensiones. Están pensados en función de los desempeños docentes que conduzcan al logro de aprendizajes fundamentales por parte de los estudiante s, aquellos aprendizajes que responden a

\section{1 un1Pé}


las exigencias de la sociedad actual.

Los aprendizajes fundamentales definidos son: ap rend er a aprender, aprender a pensar creativamente y expresarse artísticamente, aprender a actuar con eficacia transformando la realidad, aprender a convivir, aprender a pensar, aprender a afirmar su propia identidad y su autoestima.

Lo que sigue es un brevísimo extracto de las cuatro dimensiones que, a decir de Guerrero, L. (2010), "implican una visión de la docencia comprometida con la formación humana de las personas, respetuosa de sus derechos fundamentales, capaz de vincularse con ellas demostrándoles aceptación, acogida y responsabilidad, de moverse en distintos escenarios, involucrada eficientemente tanto en los procesos pedagógicos como en la construcción de un marco de políticas educativas a distinta escala":

1. Promoción de una sana convivencia para lograr aprendizajes fundamentales: desempeños que tienen que ver con la calidad de las interacciones entre docentes y estudiantes y entre los mismos alumnos, interacción con la naturaleza.

2. Conducción de los procesos de enseñanza para lograr aprendizajes fundamentales: se refieren al quehacer propiamente dicho del maestro como promotor de los aprendizajes, desempeños relacionados con sus laborares de planificación, conducción y evaluación de los procesos de enseñanza-aprendizaje.

3. Participación en la gestión y organización escolar para lograr aprendizajes fundamentales: desempeños que buscan construir una gestión educativa participativa y de calidad, una comunidad profesional de aprendizaje y la alianza escuela-comunidad.

4. Desarrollo profesional para lograr aprendizajes fundamentales: ejercicio profesional caracterizado por una actuación reflexiva y crítica, desempeños relacionados con las cualidades personales y su compromiso con su desarrollo profesional.

(El documento completo de estos criterios puede ser descargado dela página Web delCNE).

El desarrollo de un enfoque integral para evaluar a los maestros es crítico. No es una tarea fácil, en nuestro país ya se ha iniciado el camino, y de los errores se aprende. Es sumamente necesario considerar las circunstancias bajo las cuales los sistemas de evaluación del desempeño docente resultan ser más eficaces, equitativos y confiables. Es imprescindible atender aspectos como conciliar las demandas de mejores prácticas educacionales con la apropiación del proceso por parte de los maestros; definir la conveniencia de utilizar los indicadores de rendimiento escolar para medir su desempeño, como se hace en otros sistemas de evaluación docente, y atender la evaluación docente también dentro de un concepto pluricultural y multílingüe, así como aumentar el decisivo atractivo y reconocimiento de la profesión docente, entre otros.

En resumen, es necesario establecer con precisión estándares de práctica docente, que permitan definir políticas de formación profesional, así como realizar la evaluación docente de manera sistemática y coherente. Existen muchos beneficios del uso de los estándares de práctica docente. Creemos que los más importantes son los beneficios internos: es decir, cuando los docentes saben lo que se espera de ellos pueden comprometerse en una autoevaluación, una reflexión sobre su práctica y un diálogo profesional estructurado en torno a los estándares, en un proceso queles permitirá también valorar su profes ión.

Las naciones más institucionalizadas impulsan desde hace varios años experiencias de premiación a la excelencia docente. Países como Australia, Estados Unidos, Canadá, España y el Reino Unido desarrollan numerosas instancias de premiación a los mejores docentes, y todas ellas comparten la misma preocupación por elevar la valoración de la profesión docente. El instrumento privilegiado es el reconocimiento a un número reducido de maestros y profesores que, por sus cualidades y efectividad en el ejercicio de la profesión, pueden considerarse casos inspiradores para el resto del colectivo docente y para la sociedad en general.

Igualmente, en algunos países de América Latina existen iniciativas de reconocimiento de la docencia efectiva que no necesariamente son implementadas por organismos del Estado, como en el caso del Perú, con el reconocimiento denominado "Maestro que deja huella", auspiciado por una entidad bancaria. Otras iniciativas son el Premio Educador Nota 10 en Brasil; el Premio Compartir al Maestro en Colombia; el Premio Maestro 100 Puntos en Guatemala y el Premio ABC en México. Estos premios se instalan como mecanismos interesantes y complementarios a otras políticas que apuestan a mejorar la efectividad del desempeño de 10 docentes. Una tarea necesaria es la de sistematizar la experiencia peruana de 
cuatro años a la fecha, a fin de identificar las características comunes que comparten estos maestros ganadores de estos premios, que hacen que su desempeño sea eficiente.

\section{Evaluación institucional}

Para promover la calidad en la formación de estudiantes es importante que la institución educativa desarrolle una gestión de calidad dentro de una cultura de calidad. En esta última, deben estar involucrados todos los miembros de la comunidad educativa, docentes, alumnos, personal docente y administrativo, incluyendo padres de familia; todos debenidentificarse con estafilosofía y practicarla en su quehacerdiario.

En el pasado, muchas instituciones educativas, luego de serias reflexiones respecto a la naturaleza de su labor, ¿qué hacen, cómo lo hacen, y por qué y para quienes lo hacen?, implementaron procesos de planeamiento estratégico con el objetivo de mejorar su gestión y visualizar su futuro. Entre los objetivos de este proceso estaba la construcción de su misión y visión, algo que los conduciría a lograr la calidad del servicio ofrecido. Estos procesos, conjuntamente con las certificaciones ISO, se trasladaron de las empresas a lasinstituciones educativas.

Estas prácticas de evaluación interna llevaron a identificar algunos puntos importantes que la institución educativa debía promover responsablemente para conseguir resultados de aprendizaje de calidad. Entre éstos están la institucionalización del trabajo en equipo, donde alumnos, maestros y directivos trabajen conjuntamente, donde la práctica del aprendizaje colaborativo y la autoevaluacíón se desarrollan en forma permanente y donde la toma de decisiones sea compartida. Lo que estos aspectos indican es la práctica deuna organización más horizontal.

Estas escuelas, bajo una nueva gestión, procurarán reformas en relación al tiempo y al espacio. Se rompen los horarios convencionales que no permiten el desarrollo de actividades como la investigación, experimentación, el debate de ideas, la atención a las diferencias, etc., y se extiende el concepto de aula a la comunidad, permitiendo que los alumnos se pongan en contacto con los procesos vivos de la cultura y lo que se produce en ella. Estos cambios abren nuevas posibilidades de desempeño docente y, por supuesto, nuevas estrategias de aprendizajes y de evaluación.

\section{El Proyecto Educativo Nacional considera,}

igualmente, dentro de sus objetivos estratégicos la necesidad de promover la mejora de la gestión escolar; particularmente en el nivel de Educación Básica Regular. Desafortunadamente, el cambio sustantivo dela gestión educativa, en el caso delas instituciones públicas, está amarrada a procedimientos administrativos ele los órganos oficiales; ele ahí la postura del CNE por reorganizar la estructura, funciones y competencias ele los tres niveles ele gobierno: el nacional, regional y local, dotando ele autonomía ele gestión al centro educativo.

Algunas instituciones educativas, preocupadas por los resultados ele los aprendizajes de sus estudiantes, así como en respuesta a procesos ele benchmarking inducidos por el mercado educativo global y la imagen institucional, han desarrollado también, aunque en menor escala, evaluaciones internas ele todos sus procesos pedagógicos y aclmínístratívos, ya sea con diseños propios o asesorías puntuales con un enfoque ele autoevaluacíón. La evaluación institucional, desarrollada en forma interna por las instituciones educativas con el propósito ele mejorar sus servicios y mantenerse en el ranking, ha dado paso al concepto de acreditación externa por instituciones especialmente identificadas o propuestas paratalfin.

La acreditación es un proceso ele comprobación de la calidad del servicio educativo que ofrece una determinada organización o institución, y que se determina teniendo en cuenta ciertos parámetros o estándares. Estos explicitan las mejores prácticas en los diversos componentes ele la actividad educativa: desde la actuación del profesor en el aula y los resultados ele los aprendizajes ele los estudiantes, actividades de innovación o investigación, hasta la existencia y uso ele recursos, infraestructura y equipamiento educativos, pasando por la gestión administrativa y la participación ele la comunidad educativa, entre otros. En suma, lo que se busca es comprobar la calidad educativa que ofrece la institución en términos de aprendizajes ele los alumnos, desempeño ele los profesores, procesos que realizan, recursos disponibles, etc. Así, luego ele un proceso ele autoevaluacíón realizada por la misma institución, siguiendo lo prescrito por el modelo adoptado libremente, o exigido y confirmado por la Evaluación Externa, el órgano acreditador, nacional o internacional, determina sí la escuela o institución educativa puede ser considerada como una institución de calidad. La percepción de calidad puede variar dependiendo de factores internos importantes para la institución, o externos

\section{1 un1Fé}


requeridos o percibidos por el usuario del servicio educativo ola comunidad en general.

En el Perú, el año 2006 se crea el SINEACE -Sistema Nacional de Evaluación, Acreditación y Certificación de la Calidad Educativa- con el objetivo de garantizar que todos los estudiantes ejerzan su derecho a una educación de calidad. Según este dispositivo, todas las instituciones del sistema educativo deben evidenciar niveles básicos de calidad como resultado de un proceso de acreditación basado en determinados estándares e indicadores. Entre sus funciones están los siguientes: contribuir a la medición y evaluación de los aprendizajes en el sistema educativo; contribuir a una mejora de la calidad de los servicios educativos en todas las etapas, niveles, modalidades, formas, ciclos, programas e instituciones del país; acreditar instituciones y programas educativos y certificar competencias laborales y profesionales; asegurar a la sociedad que las instituciones educativas que forman parte del sistema educativo cumplen los requisitos decalidad.

Dentro del SINEACE existen órganos operadores de la acreditación para los distintos niveles y modalidades del sistema educativo. Cada uno de ellos, desde metodologías diferentes, ha elaborado sus propios estándares a fin de asegurar su aplicación en las mejores condiciones y plazos. El IPEBA

-Instituto Peruano de Evaluación, Acreditación y Certificación de la Calidad de la Educación Básicaes el órgano encargado de acreditar a todas las instituciones educativas del país en Educación Básica Regular y Técnico-Productiva. De igual manera, el CONEAU es el órgano encargado de acreditar a las instituciones de Educación Superior Universitaria (pre -grado y posgrado), así como lo hace el CONEACES con las instituciones de educación superior no universitaria. Estos organismos han publicado los estándares e indicadores correspondientes en cada uno de los niveles y modalidades, algunos de los cuales se pueden descargarvirtualmente.

El propósito fundamental de los procesos de acreditación, cualquiera sea el nivel o modalidad educativa, eselmejoramiento delacalidadeducativa.

Los procesos de evaluación planteados por estos organismos operadores son autoevaluación, evaluación externa y a cr ed i t ac ió $\mathrm{n}$. La autoevaluación es efectuada por la propia institución bajo los indicadores que el organismo acreditador señala. Esta es una evaluación interna de todos los aspectos que conforman el servicio educativo. La evaluación externa es efectuada por una comisión externa nombrada por el órgano acreditador, e integrada por profesionales especialmente preparados y reconocidos para esta tarea. Finalmente, la acreditación es otorgada como resultado del análisis y correspondencia de los dos procesos previos deevaluación.

Las acreditaciones son voluntarias en el nivel de Educación Básica. En la Educación Superior, la acreditación es obligatoria para las carreras de Ciencias de la Salud, Educación y Derecho, y voluntaria para las demás carreras. El CONEAU prioriza la acreditación de carreras profesionales y estudios de posgrado, antes de proceder a evaluaciones institucionales.

En razón de estar moviéndonos en un mundo sumamente cambiante, glob al , dinámico y competitivo, existe en la actualidad, por parte de algunas instituciones públicas y privadas delnivelde Educación Superior y de Educación Básica, una especial motivación de mejor amiento de sus procesos educativos y administrativos orientados a conseguir la tan ansiada calidad educativa.

Existe a nivel mundial una serie de organizaciones acreditadoras muy reconocidas, con modelos , procesos y exigencias de acreditación diferentes. Asimismo, en América Latina y El Caribe, numerosas experiencias de acreditación se vienen desarrollando desde principios de los años' 90, particularmente en Educación Superior; algunas implementadas desde el Estado como mandato oficial y otras a través de organismos privados. En ambos casos, estos procesos se dan en respuesta a las necesidades de las instituciones educativas de transformación y adaptación a las exigencias nacionales y globalizantes, así como a la demanda de garantía y rendición de cuentas por parte del Estado y de la sociedad.

En el Perú, el nivel de Educación Superior, con mayor énfasis que en la Educación Básica, muchas instituciones vienen realizando procesos de acreditación con organismo s nacionales y extran jeros. ElICACIT, organismo nacional creado el 2003, utilizando la metodología del ABET

-Acrcditation Board far Enginccring and Tcchnology- de Estados Unidos, acredita carreras profesionales de Computación, Ingeniería y Tecnología (Valdés, 2009).

A la fecha, el SINEACE no ha acreditado aún a 
ninguna institución. Por haber serias observaciones a los estándares elaborados por este organismo, particularmente por el CONEAU, algunas universidades han recurrido a las acreditaciones extranjeras. Los cuestionamientos que han sido señalados están básicamente relacionados con la inconsistencia y falta de pertinencia de los estándares, problemas en su concepción y formulación. Estos estándares se presentan como un listado prescriptivo, uniformizador y rígido.

Las acreditaciones internacionales, particularmente las latinoamericanas, son por lo general muy efectivas y están de acuerdo con las realidades locales e internacionales. Mantienen el objetivo fundamental de la mejora de la calidad y cuentan con una experiencia razonable en el proceso de acreditación deuniversidades.

Finalmente, debe mencionarse que el Presidente dela República ha señalado que para hacer frente a la proliferación de instituciones de educación superior, se establecerán mecanismos para que los títulos a nombre de la nación sean dados por universidades e institutos de educación superior debidamente acreditados. El énfasis estará en la acreditación y no en los títulos, ya que, para los empleadores, antes que el título profesional se valora más la calidad de la institución donde el profesional cursó sus estudios.

\section{REFERENCIAS}

Alfaro, M. (2000). Evaluación del aprendizaje. Maracaibo: IUPEL Bordas, l.; Cabrera, F. (2001). Estrategias de evaluación de los aprendizajes centrados en el proceso. Revista Española de Pedagogía. Enero - abril 2001. España.

Campos, A. (2006). Mapas conceptuales, mapas mentales y otras formas derepresentacióndelconocimiento. Bogotá:Ed. Magisterio.

Consejo Nacional de Educación (2007). Proyecto Educativo Nacional al 2021: la educación que queremos para el Perú. Lima: Ministerio de Educación - C NE.

Consejo Nacional de Educación. (2009). Reglamento del Sinease Para comprobar lacalidad educativa? Lima:CNE Opina.Boletín $\mathrm{N}^{\circ} 6$.

Consejo Nacional de Educa ción - Mesa Interinstitucional (2011). Ser Maestro en el Pení: hacia una propuesta de criterios de buen desempeño docente. II Congreso Pedagógico Nacional. Lima. Documento detrabajo.

Danielson, C. (2007). Enhancing professional practice: a framework for teaching. 2nd ed. Alexandria, Virginia: Association for Supervision and Currículum Development (ASCD).

Delors, J. (ed) (1996). La educación encierra un tesoro. Madrid: Santillana - UNESCO.

Farr, R.; B. TONE (1994). Portafolio. Performance asessment. Helping students evaluate their progress as readers and writers. For Worth: Harcourt Brace CollegePublishers.

Guerrero, L.(2011).Propuesta de criterios de buena docencia. Segundo Congreso Pedagógico Nacional: "Ser maestro en el Perú...". Lima. Documento de trabajo.

Isoré, M. (2009). Teacher evaluation: current practices in OECD. Countries and a literature review. París: OECD. Education WorkingPaperNº23.
Ministerio de Educación (2011). Resultados de la Evaluación Censal del 2010. lima: Vice -Ministerio de Gestión Pedagógica. Presentación alCNE.

Mueller,J. (2005). The authentic assessment toolbox: enhancing student learning through online faculty development. MERLOT - Journal of Online LearningandTeaching. Vol. l, $\mathrm{N}^{\circ} \mathrm{l}$.

Novak, D. A.; Gowin, D. B. (1988). Aprendiendo a aprender. Barcelona: Martínez Roca.

Quaas, C. (2000). Nuevos enfoques en la evaluación de los aprendizajes. Revista Enfoques Educacionales. Vol. 2, No 2. Universidad de Chile.

Rivera, J. L. (2004). El aprendizaje significativo y la evaluación de los aprendizajes. Revista de Investigación Educativa, Año 8, № 1 IIE-FE-UNMSM.

Roman, M. (2010). La voz ausente de estudiantes y padr es en la evaluación del desempeño docente. PREAL Serie documentos. $\mathrm{N}^{\circ}$ 49. Chile.

SACS. (2009). Southern Association of Colleges and Schools. Accreditation Process.

SINEACE. (2008). Acreditación de la carrera profesional de Educación: Sineace, Coneau, antecedentes ylegislación. Seminari o-taller.

Vaillant,D. Rosell,C.(2010). Elreconocimientodela docencia efectiva: Premioa la excelencia. PREAL Seriedocumentos $\mathrm{N}^{\circ} 48$. Chile.

Valdés, H. (2009). Manual de buenas prácticas de evaluación del desempeño profesional de los docentes. Consejo Nacional de Educación.

UNESCO (2007). Evaluación del desempeño y carrera profesional docente: una panorámica de América y Europa. Oficina Regional de Educación para América Latina y el Caribe. Santiago, Chile: UNESCO.

Guadalupe Méndez Zamalloa, Ph. D.

Educadora, magíster y doctora en Educación por Florida State University, cuenta con un diplomado en Administración Educativa. Ha sido directora de la Escuela de Empresarios del Instituto Peruano de Administración de Empresa (IPAE) y docente en las Universidades de Lima, UNIFÉ y Cayetano Heredia. Además, es Miembro del Consejo Nacional de Educación. 